(n)

trobertivier Journal of Nonlinear Mathematical Physics

\title{
Proper rational and analytic first integrals for asymmetric 3- dimensional Lotka-Volterra systems
}

Jaume Llibre, Clàudia Valls

To cite this article: Jaume Llibre, Clàudia Valls (2017) Proper rational and analytic first integrals for asymmetric 3-dimensional Lotka-Volterra systems, Journal of Nonlinear Mathematical Physics 24:3, 393-404, DOI:

https://doi.org/10.1080/14029251.2017.1341701

To link to this article: https://doi.org/10.1080/14029251.2017.1341701

Published online: 04 January 2021 


\title{
Proper rational and analytic first integrals for asymmetric 3-dimensional Lotka-Volterra systems
}

\author{
Jaume Llibre \\ Departament de Matemàtiques, Universitat Autònoma de Barcelona, \\ 08193 Bellaterra, Barcelona, Catalonia, Spain \\ jllibre@mat.uab.cat \\ Clàudia Valls \\ Departamento de Matemática, Instituto Superior Técnico, \\ 1049-001 Lisboa, Portugal \\ cvalls@math.ist.utl.pt
}

Received 12 February 2017

Accepted 20 April 2017

\begin{abstract}
We go beyond in the study of the integrability of the classical model of competition between three species studied by May and Leonard [19], by considering a more realistic asymmetric model. Our results show that there are no global analytic first integrals and we provide all proper rational first integrals of this extended model by classifying its invariant algebraic surfaces.

Keywords: polynomial integrability; rational integrability; analytic integrability; asymmetric Lotka-Volterra systems.
\end{abstract}

2000 Mathematics Subject Classification: 34C05, 34A34, 34C14

\section{Introduction and statement of the main results}

Nonlinear differential equations govern many branches of applied mathematics, physics and sciences in general. A 3-dimensional system with two first integrals whose gradients are linearly independent in $\mathbb{R}^{3}$ (except perhaps in a zero Lebesgue measure set) is completely solvable in the sense that the intersections of the invariant levels of these two first integrals determine the trajectories of the system. On the other hand, the knowledge of only one first integral does not determine completely the phase portrait of the system but reduces the study of its dynamics by one dimension (i.e. from dimension 3 to dimension 2). Therefore knowing whether there exists a first integral is important in the qualitative theory of differential equations. Different methods exist for studying the existence of first integrals of non-linear ordinary differential equations relying on: the well-known Darboux theory of integrability [8,18], the so-called Noether symmetries [5], the so-called Lie symmetries [1,24], the well-known Painlevé analysis [3], the use of Lax pairs [12], the so-called direct method $[9,10]$, the well-known Carleman embedding procedure $[2,6]$, the so-called linear compatibility analysis method [25], etc.

In the present paper using the well-known Darboux theory of integrability we determine the existence of first integrals in some given classes for the following asymmetric model that studies the competition among three species and that is the asymmetrization of the initial model used by May and Leonard [19]. This asymmetric model is 


$$
\begin{aligned}
\dot{X} & =X\left(1-X-a_{1} Y-b_{1} Z\right), \\
\dot{Y} & =Y\left(1-b_{2} X-Y-a_{2} Z\right), \\
\dot{Z} & =Z\left(1-a_{3} X-b_{3} Y-Z\right),
\end{aligned}
$$

where

$$
0<a_{i}<1<b_{i} \quad \text { for } i=1,2,3
$$

and the dot denotes derivative with respect to the time $t$. Model (1.1) was studied by Chi, Hsu and Wu in [7] and it controls the competition between three species with the same intrinsic growth rates and different competition coefficients. From the results on a two-dimensional competitive system [26], the assumption $0<a_{i}<1<b_{i}$ for $i=1,2,3$ ensures that there is an orbit on the $x y$-plane connecting the equilibrium $(0,1,0)$ to the equilibrium $(1,0,0)$, an orbit on the $x z$-plane connecting the equilibrium $(1,0,0)$ to the equilibrium $(0,0,1)$, and an orbit on the $y z$-plane connecting the equilibrium $(0,0,1)$ to the equilibrium $(0,1,0)$. May and Leonard [19] were the first to study the symmetric case that is, when $a_{1}=a_{2}=a_{3}$ and $b_{1}=b_{2}=b_{3}$.

The symmetric case was studied intensively from the view point of the dynamics by several authors including May and Leonard themselves and from the view of of the integrability by Leach and Miritzis in [13] (obtaining partial results) and by Llibre and Valls in [16] characterizing completely the polynomial, rational and analytic first integrals. The asymmetric model, being much more realistic, has been studied from the dynamical point of view in [7]. There the authors proved the asymptotic stability of a stationary point in certain parameter regions, which precludes the existence of a global analytic first integral. In this paper we study the polynomial, rational and global analytic first integrals for all the values of the parameters.

In order to study the integrability of system (1.1) we first do the change of variables

$$
x=X e^{-t}, \quad y=Y e^{-t}, \quad z=Z e^{-t}, \quad s=e^{t},
$$

and system (1.1) becomes

$$
\begin{aligned}
& x^{\prime}=-x\left(x+a_{1} y+b_{1} z\right), \\
& y^{\prime}=-y\left(b_{2} x+y+a_{2} z\right), \\
& z^{\prime}=-z\left(a_{3} x+b_{3} y+z\right),
\end{aligned}
$$

where $0<a_{i}<1<b_{i}$ for $i=1,2,3$, and the prime denotes derivative with respect to $s$.

More precisely, we will completely characterize the polynomial, rational and analytic first integrals of system (1.2). To do so we introduce some notation. We set 


$$
\begin{aligned}
G & =z+\frac{\left(1-a_{3}\right)}{b_{1}-1} x+\frac{\left(a_{1}-1\right)\left(a_{3}-1\right)}{\left(b_{1}-1\right)\left(b_{2}-1\right)} y, \\
b_{3}^{*} & =1+\frac{\left(1-a_{1}\right)\left(1-a_{2}\right)\left(1-a_{3}\right)}{\left(b_{1}-1\right)\left(b_{2}-1\right)}, \\
k_{1}^{*} & =\frac{b_{1}-1}{1-a_{2}}>0, \\
k_{2}^{*} & =\frac{\left(b_{1}-1\right)\left(b_{2}-1\right)}{\left(1-a_{2}\right)\left(1-a_{3}\right)}>0, \\
k_{3}^{*} & =\frac{1-a_{2}+a_{2} a_{3}-a_{3} b_{1}-b_{2}+b_{1} b_{2}}{\left(1-a_{2}\right)\left(1-a_{3}\right)}>0 .
\end{aligned}
$$

Let $U \subset \mathbb{R}^{3}$ be an open subset and consider the vector field

$$
\mathscr{X}=-x\left(x+a_{1} y+b_{1} z\right) \frac{\partial}{\partial x}-y\left(b_{2} x+y+a_{2} z\right) \frac{\partial}{\partial y}-z\left(a_{3} x+b_{3} y+z\right) \frac{\partial}{\partial z},
$$

on $U$ related with system (1.2). A non-constant function $H: U \rightarrow \mathbb{R}$ is a first integral of $\mathscr{X}$ if $H(x(t), y(t), z(t))$ is constant for all values for which the solution $(x(t), y(t), z(t))$ of $\mathscr{X}$ is defined on $U$.

$H$ is a polynomial first integral if $H$ is a polynomial, $H$ is a proper rational first integral if $H$ is a rational function which is not a polynomial and $H$ is an analytic first integral if $H$ is an analytic function.

The symmetric case, i.e., when $a_{1}=a_{2}=a_{3}=a$ and $b_{1}=b_{2}=b_{3}=b$ the polynomial, rational and analytic integrability was studied in [16] where the authors proved the following result.

Theorem 1.1. For the differential system (1.2) with $a_{1}=a_{2}=a_{3}=a$ and $b_{1}=b_{2}=b_{3}=b$, the following statements hold.

(a) There are no polynomial first integrals.

(b) The unique proper rational first integrals are rational functions in the variable

$$
\frac{x y z}{(x+y+z)^{3}}
$$

whenever $a+b=2$.

(c) There are no global analytic first integrals.

The main result of this paper is the following one.

Theorem 1.2. For the differential system (1.2) the following statements hold.

(a) There are no polynomial first integrals.

(b) If $b_{3}=b_{3}^{*}$ then

$$
\frac{x y^{k_{1}^{*}} z^{k_{2}^{*}}}{G^{k_{3}^{*}}}
$$

is a first integral. Additionally, if $k_{1}^{*}, k_{2}^{*}, k_{3}^{*} \in \mathbb{N}$, this first integral is the unique proper rational first integral of the system. 
(c) There are no global analytic first integrals.

Note that Theorem 1.2 reduces to Theorem 1.1 when $a_{1}=a_{2}=a_{3}=a$ and $b_{1}=b_{2}=b_{3}=$ $b$. Therefore in the proof of Theorem 1.2 we will only consider the case in which $\left(a_{1}-a_{2}\right)^{2}+$ $\left(a_{1}-a_{3}\right)^{2}+\left(a_{2}-a_{3}\right)^{2} \neq 0$ and $\left(b_{1}-b_{2}\right)^{2}+\left(b_{1}-b_{3}\right)^{2}+\left(b_{2}-b_{3}\right)^{2} \neq 0$. The integrability of other 3-dimensional Lotka-Volterra systems other than system (1.2) has been studied, see for instance $[4,11,15,20-22]$ and the references therein. We must remark that under the condition $b_{3}=b_{3}^{*}$ the differential system (1) exhibits a Hopf bifurcation and a family of neutrally stable periodic orbits, see for a proof [7]; while under the same condition system (2) and system (1) have the first integral given in statement (b) of Theorem 1.2.

Note that since system (1.2) is homogeneous, the knowledge of its homogeneous polynomial first integrals determine easily all its polynomial and its analytic first integrals (see Proposition 2.3 for further details). With this observation it is clear that statement (c) of Theorem 1.2 follows from statement (a) (of the same theorem).

In the proof of Theorem 1.2 we will use the invariant algebraic surfaces of system (1.2). This is precisely on what is based the Darboux theory of integrability which was introduced by Darboux [8] in 1878. This theory is valid for either real or complex polynomial differential equations and sometimes the knowledge of complex invariant algebraic curves is necessary for obtaining all the real first integrals of a real polynomial differential equation (see for instance [14]).

We say that $h=h(x, y, z) \in \mathbb{C}[x, y, z] \backslash \mathbb{C}$ is a Darboux polynomial of system (1.2) if it satisfies $\mathscr{X} h=K h$ for some $K=K(x, y, z) \in \mathbb{C}[x, y, z]$. The polynomial $K$ is called the cofactor of $h$ and has degree at most 1 . In this case we also say that $h=0$ is an invariant algebraic curve of the vector field $\mathscr{X}$ associated to system (1.2) and $K$ is also called the cofactor of $h=0$. Throughout the paper when we say that a polynomial is irreducible, we mean irreducible in $\mathbb{C}[x, y, z]$. Moreover, we recall that the polynomial first integrals are the Darboux polynomials with zero cofactor.

Theorem 1.3. The unique irreducible Darboux polynomials with non-zero cofactor of system (1.2) are $x, y, z$ with cofactors $-\left(x+a_{1} y+b_{1} z\right),-\left(b_{2} x+y+a_{2} z\right)$ and $-\left(a_{3} x+b_{3} y+z\right)$ respectively, for all $a_{i}, b_{i} \in \mathbb{R}$ for $i=1,2,3$; and $G$ whenever $b_{3}=b_{3}^{*}$ (see (1.3)) with cofactor $-x-y-z$.

In Section 2 we state and prove some auxiliary results of system (1.2) that will be used in the paper. In Section 3 we prove some results of system (1.2) restricted to either $x=0$, or $y=0$, or $z=0$ and that will play an important role in the proof of Theorem 1.2. Finally, in section 4 we prove Theorem 1.3, and in section 5 we prove Theorem 1.2.

\section{Preliminaries}

Proposition 2.1. Let $f \in \mathbb{C}[x, y, z]$ and $f=\prod_{j=1}^{s} f_{j}^{\alpha_{j}}$ be its decomposition into irreducible factors in $\mathbb{C}[x, y, z]$. Then $f$ is a Darboux polynomial of system (1.2) if and only if $f_{j}$ are Darboux polynomials of system (1.2) for $j=1, \ldots, j$. Additionally, if $K$ and $K_{j}$ are the cofactors of $f$ and $f_{j}$ respectively, then $K=\sum_{j=1}^{s} \alpha_{j} K_{j}$.

Proof. See [14].

The following three results are well-known and can be proved easily using Darboux theory of integrability (for the second one see also [23] and for the third one see [17]). 
Proposition 2.2. The homogeneous polynomial differential system (1.2) has a proper rational first integral if and only if it has two Darboux polynomials with the same non-zero cofactor.

Lemma 2.1. Any Darboux polynomial $f \neq 0$ of the homogeneous polynomial differential system (1.2) has a cofactor of the form

$$
K=\alpha_{1} x+\alpha_{2} y+\alpha_{3} z
$$

with $\alpha_{i} \in \mathbb{C}, i=1,2,3$.

Proposition 2.3. The following statements hold:

(a) Let $f$ be a polynomial and write it in sum of its homogeneous parts as $f=\sum_{j=1}^{n} f_{j}$. Then $f$ is a Darboux polynomial of the homogeneous polynomial differential system (1.2) with cofactor $K$ if and only if $f_{j}$ is a Darboux polynomial of homogeneous polynomial differential system (1.2) with cofactor $K$ for $j=1, \ldots, n$.

(b) Let $f$ be a formal power series and write it in sum of its homogeneous parts as $f=\sum_{j \geq 1} f_{j}$, with $f_{j}$ being homogeneous polynomials of degree $j$. Then $f$ is a formal first integral of the homogeneous polynomial differential system (1.2) if and only if $f_{j}$ is a polynomial first integral of the homogeneous polynomial differential system (1.2) for all $j \geq 1$.

Proposition 2.4. The unique irreducible Darboux polynomials of degree 1 with non-zero cofactor of system (1.2) are: $x, y, z$ with cofactors $-\left(x+a_{1} y+b_{1} z\right),-\left(b_{2} x+y+a_{2} z\right)$ and $-\left(a_{3} x+b_{3} y+z\right)$ respectively, for all $a, b \in \mathbb{R}$ and $G$ whenever $b_{3}=b_{3}^{*}$ (see (1.3)) with cofactor $-x-y-z$.

Proof. It follows easily from direct computations and the definition of Darboux polynomial.

3. System (1.2) restricted to $x=0$, or $y=0$, or $z=0$.

We will consider in this section system (1.2) restricted to either $x=0$, or $y=0$, or $z=0$.

Theorem 3.1. For system (1.2) restricted to $z=0$ the following statements hold.

(a) There are no homogeneous polynomial first integrals.

(b) All the irreducible Darboux polynomials with non-zero cofactor are $x$ and $y$ for all $0<$ $a_{1}<1<b_{2}$; and additionally $\left(b_{2}-1\right) x+\left(1-a_{1}\right) y$.

Proof. First we consider system (1.2) restricted to $z=0$, that is,

$$
\dot{x}=-x\left(x+a_{1} y\right), \quad \dot{y}=-y\left(b_{2} x+y\right) .
$$

It follows by direct computations that

$$
H=x^{1-b_{2}} y^{1-a_{1}}\left(\left(b_{2}-1\right) x+\left(1-a_{1}\right) y\right)^{-1+a_{1} b_{2}}
$$

is a first integral of system (3.1). Furthermore, it is a homogeneous polynomial of degree $n \geq 1$ if and only if

$$
1-a_{1}=n_{1}, \quad 1-b_{2}=n_{2}, \quad a_{1} b_{2}-1=n-n_{1}-n_{2}, \quad n_{1}+n_{2} \leq n, \quad n_{1}, n_{2} \in \mathbb{N},
$$

or equivalently, if and only if,

$$
1-a_{1}=n_{1}, \quad 1-b_{2}=n_{2}, \quad n_{1} n_{2}=n, \quad n_{1}+n_{2} \leq n, \quad n_{1}, n_{2} \in \mathbb{N},
$$

which is not possible because $0<a_{1}<1$ and $b_{2}>1$. Hence statement (a) is proved. 
It is also straightforward by direct computations that the unique irreducible Darboux polynomials of system (3.1) are $x$ and $y$ and $\left(b_{2}-1\right) x+\left(1-a_{1}\right) y$ (note that $a_{1} \neq 1, b_{2} \neq 1$ and that irreducible homogeneous polynomials in two variables are necessarily of degree one).

To complete the proof of the proposition we will proceed by contradiction. Let $f$ be an irreducible homogeneous Darboux polynomial of system (3.1) of degree $n \geq 2$ and so it satisfies

$$
-x\left(x+a_{1} y\right) \frac{\partial f}{\partial x}-y\left(b_{2} x+y\right) \frac{\partial f}{\partial y}=\left(\alpha_{1} x+\alpha_{2} y\right) f, \quad \alpha_{1}, \alpha_{2} \in \mathbb{C} .
$$

First we assume that $\alpha_{1} \neq 0$ or $\alpha_{2} \neq 0$. If we restrict equation (3.2) to $x=0$ and denote the restriction of $f$ to $x=0$ by $\bar{f}$, then $\bar{f}=\bar{f}(y) \neq 0$ (otherwise $f$ would be reducible) is a homogeneous polynomial of degree $n$. We write it as $\bar{f}=\beta_{0} y^{n}$ with $\beta_{0} \in \mathbb{C} \backslash\{0\}$. Clearly $\bar{f}$ satisfies

$$
-y^{2} \frac{d \bar{f}}{d y}=\alpha_{2} y \bar{f} \text {. }
$$

So $\bar{f}=\alpha_{0} y^{-\alpha_{2}}$ with $\alpha_{0} \in \mathbb{C} \backslash\{0\}$. Now equating the two expressions for $\bar{f}$ we get $\alpha_{2}=-n$. In a similar manner restricting to $y=0$ we get that $\alpha_{1}=-n$. Thus $K=-n(x+y)$ and equation (3.2) becomes

$$
-x\left(x+a_{1} y\right) \frac{\partial f}{\partial x}-y\left(b_{2} x+y\right) \frac{\partial f}{\partial y}=-n(x+y) f .
$$

Since $b_{2} \neq 1$ and $a_{1} \neq 1$, we introduce the change of variables $(X, Y)=\left(\left(b_{2}-1\right) x+\left(1-a_{1}\right) y, y\right)$. In these new variables system (3.1) becomes

$$
X^{\prime}=\frac{X}{1-b_{2}}\left(X+\left(a_{1}+b_{2}-2\right) Y\right), \quad Y^{\prime}=\frac{Y}{1-b_{2}}\left(b_{2} X+\left(a_{1} b_{2}-1\right) Y\right) .
$$

We have that $\tilde{f}=\tilde{f}(X, Y)=f(x, y)$ satisfies

$$
\begin{aligned}
& \frac{X}{1-b}\left(X+\left(a_{1}+b_{2}-2\right) Y\right) \frac{\partial \tilde{f}}{\partial X}+\frac{Y}{1-b_{2}}\left(b_{2} X+\left(a_{1} b_{2}-1\right) Y\right) \frac{\partial \tilde{f}}{\partial Y} \\
& =\frac{n}{1-b_{2}}\left(X+\left(b_{2}+a_{1}-2\right) Y\right) \tilde{f} .
\end{aligned}
$$

Let now $\hat{f}$ be the restriction of $\tilde{f}$ to $X=0$. Since $f$ is irreducible, $\hat{f} \neq 0$, and $\hat{f}$ satisfies (3.3) restricted to $X=0$, that is

$$
\frac{\left(a_{1} b_{2}-1\right) Y^{2}}{1-b_{2}} \frac{d \hat{f}}{d Y}=\frac{n}{1-b_{2}}\left(b_{2}+a_{1}-2\right) Y \hat{f} .
$$

Solving this linear differential equation we obtain $\hat{f}=\beta_{0} y^{n\left(2-a_{1}-b_{2}\right) /\left(1-a_{1} b_{2}\right)}$, with $\beta_{0} \in \mathbb{C} \backslash\{0\}$. Since $\hat{f}$ has degree $n$, we must have $2-a_{1}-b_{2}=1-a_{1} b_{2}$, or equivalently $\left(a_{1}-1\right)\left(b_{2}-1\right)=0$. That is $a_{1}=1$ or $b_{2}=1$, a contradiction. This completes the proof.

Theorem 3.2. For system (1.2) restricted to $x=0$ the following statements hold.

(a) There are no homogeneous polynomial first integrals.

(b) All the irreducible Darboux polynomials with non-zero cofactor are $x$ and $y$ for all $0<$ $a_{2}<1<b_{3}$; and additionally $\left(b_{3}-1\right) x+\left(1-a_{2}\right) y$.

Theorem 3.3. For system (1.2) restricted to $y=0$ the following statements hold. 
(a) There are no homogeneous polynomial first integrals.

(b) All the irreducible Darboux polynomials with non-zero cofactor are $x$ and $y$ for all $0<$ $a_{3}<1<b_{1}$; and additionally $\left(b_{1}-1\right) x+\left(1-a_{3}\right) y$.

Theorems 3.2 and 3.3 are proved in the same way as Theorem 3.1.

\section{Proof of Theorem 1.3}

In order to prove Theorem 1.3 we first study the irreducible homogeneous Darboux polynomials of degree $n \geq 2$ of system (1.2) with non-zero cofactor. This is the content of the following theorem.

Theorem 4.1. System (1.2) has no irreducible homogeneous Darboux polynomials of degree $n \geq 2$ with non-zero cofactor.

Proof. Let $f$ be a homogeneous irreducible Darboux polynomial of degree $n \geq 2$ with non-zero cofactor. In view of Lemma 2.1 we can assume that $K=\alpha_{1} x+\alpha_{2} y+\alpha_{3} z$ with $\left(\alpha_{1}, \alpha_{2}, \alpha_{3}\right) \in \mathbb{C}^{3} \backslash$ $\{(0,0,0)\}$.

We shall prove that $K=-n(x+y+z)$. By Theorem 3.1 we have that $\tilde{f}=f(x, y, 0)=$ $c x^{m_{1}} y^{m_{2}}\left(\left(b_{2}-1\right) x+\left(1-a_{1}\right) y\right)^{n-m_{1}-m_{2}}$ with $m_{1}, m_{2} \geq 0$ and then, by Proposition 2.1, the cofactor is

$$
K=\left(\left(1-b_{2}\right) m_{2}-n\right) x+\left(\left(1-a_{1}\right) m_{1}-n\right) y .
$$

Moreover the cofactor $K$ is also equal to $\alpha_{1} x+\alpha_{2} y$. This means that

$$
\alpha_{1}=\left(1-b_{2}\right) m_{2}-n, \quad \text { and } \quad \alpha_{2}=\left(1-a_{1}\right) m_{1}-n \text {. }
$$

Similarly, by Theorem 10 we have that $\bar{f}=f(0, y, z)=c_{1} y^{m_{3}} z^{m_{4}}\left(\left(b_{3}-1\right) x+\left(1-a_{2}\right) y\right)^{n-m_{3}-m_{4}}$ with $m_{3}, m_{4} \geq 0$ and then the cofactor is $K=\left(\left(1-b_{3}\right) m_{3}-n\right) y+\left(\left(1-a_{2}\right) m_{4}-n\right) z$. Moreover the cofactor $K$ is also equal to $\alpha_{2} y+\alpha_{3} z$. This means that

$$
\alpha_{2}=\left(1-b_{3}\right) m_{3}-n, \quad \text { and } \quad \alpha_{3}=\left(1-a_{2}\right) m_{4}-n .
$$

It follows from (4.2) and (4.3) that $\left(1-b_{3}\right) m_{3}-n=\left(1-a_{1}\right) m_{1}-n$ and so $\left(1-b_{3}\right) m_{3}=\left(1-a_{1}\right) m_{1}$ with $m_{1}, m_{3} \geq 0$. Since $b_{3}>1$ and $a_{1}<1$ the unique possibility is $m_{1}=m_{3}=0$.

Doing the same with the restriction of $f$ to $y=0$ we get that $m_{2}=m_{4}=0$. From (10) and (11) we get $\alpha_{1}=\alpha_{2}=\alpha_{3}=-n$. In short $K=-n(x+y+z)$ and $f$ satisfies

$$
-x\left(x+a_{1} y+b_{1} z\right) \frac{\partial f}{\partial x}-y\left(b_{2} x+y+a_{2} z\right) \frac{\partial f}{\partial y}-z\left(a_{3} x+b_{3} y+z\right) \frac{\partial f}{\partial z}=-n(x+y+z) f .
$$

We denote by $\tilde{f}=\tilde{f}(x, y)$ the restriction of $f$ to $z=0$. Since $\alpha_{i}=-n$ for $i=1,2,3$, it follows from the above discussion in the case that we restricted to $z=0$ that $\tilde{f}=f(x, y, 0)=c_{0} x^{m_{1}} y^{m_{2}}\left(\left(b_{1}-1\right) x+\right.$ $\left.\left(1-a_{1}\right) y\right)^{n-m_{1}-m_{2}}$ with cofactor (4.1). Therefore $m_{1}=m_{2}=0$ and so $\tilde{f}=\left(\left(b_{1}-1\right) x+\left(1-a_{1}\right) y\right)^{n}$ and $f=c_{0}((b-1) x+(1-a) y)^{n}+z g$, where $g=g(x, y, z)$ is a homogenous polynomial of degree $n-1$. Proceeding analogously for the restrictions of $f$ to $x=0$ and to $y=0$ we can write $f$ in the 
following three forms

$$
\begin{aligned}
f & =c_{0}\left(\left(b_{2}-1\right) x+\left(1-a_{1}\right) y\right)^{n}+z g_{0}=c_{1}\left(\left(b_{3}-1\right) y+\left(1-a_{2}\right) z\right)^{n}+x g_{1}, \\
& =c_{2}\left(\left(b_{1}-1\right) z+\left(1-a_{3}\right) x\right)^{n}+y g_{2},
\end{aligned}
$$

for some homogeneous polynomials $g_{0}, g_{1}, g_{2}$ of degree $n-1$. Note that $c_{i} \neq 0$ for $i=1,2,3$ otherwise $f$ would be reducible. We consider two cases.

Case 1: $b_{3} \neq b_{3}^{*}$. Evaluating (4.4) on $x=z=0$ we get

$$
c_{0}\left(1-a_{1}\right)^{n}=c_{1}\left(b_{3}-1\right)^{n} .
$$

Evaluating (4.4) on $x=y=0$ we obtain

$$
c_{1}\left(1-a_{2}\right)^{n}=c_{2}\left(b_{1}-1\right)^{n}
$$

and on $y=z=0$, we have

$$
c_{0}\left(b_{2}-1\right)^{n}=c_{2}\left(1-a_{3}\right)^{n} .
$$

From (4.5) and (4.6) we get

$$
c_{0}=c_{2} \frac{\left(b_{1}-1\right)^{n}\left(b_{3}-1\right)^{n}}{\left(1-a_{1}\right)^{n}\left(1-a_{2}\right)^{n}},
$$

and so from (4.7) using that $c_{2} \neq 0$ we must have

$$
\left(\frac{\left(b_{1}-1\right)\left(b_{2}-1\right)\left(b_{3}-1\right)}{\left(1-a_{1}\right)\left(1-a_{2}\right)}\right)^{n}=\left(1-a_{3}\right)^{n},
$$

and since all the factors are positive we obtain

$$
\frac{\left(b_{1}-1\right)\left(b_{2}-1\right)\left(b_{3}-1\right)}{\left(1-a_{1}\right)\left(1-a_{2}\right)}=1-a_{3},
$$

which yields

$$
b_{3}=1+\frac{\left(1-a_{1}\right)\left(1-a_{2}\right)\left(1-a_{3}\right)}{\left(b_{1}-1\right)\left(b_{2}-1\right)}=b_{3}^{*},
$$

which is not possible.

Case 2: $b_{3}=b_{3}^{*}$. From (1.3) we have

$$
G=z+\frac{\left(1-a_{3}\right)}{\left(b_{1}-1\right)\left(b_{2}-1\right)}\left(\left(b_{2}-1\right) x+\left(1-a_{1}\right) y\right),
$$

and from (4.4) we get

$$
f=c_{0}\left(\left(b_{2}-1\right) x+\left(1-a_{1}\right) y\right)^{n}+z g_{0}=\tilde{c}_{0}\left(\frac{\left(1-a_{3}\right)}{\left(b_{1}-1\right)\left(b_{2}-1\right)}\right)^{n}\left(\left(b_{2}-1\right) x+\left(1-a_{1}\right) y\right)^{n}+z g_{0},
$$

where

$$
\tilde{c}_{0}=c_{0}\left(\frac{\left(1-a_{3}\right)}{\left(b_{1}-1\right)\left(b_{2}-1\right)}\right)^{-n}
$$


Moreover, using the Newton's binomial formula we can also write

$$
f=\tilde{c}_{0} G^{n}+z g_{1},
$$

for some homogeneous polynomial $g_{1}$ of degree $n-1$. Indeed, note that

$$
\begin{aligned}
G^{n}= & \left(z+\frac{\left(1-a_{3}\right)}{\left(b_{1}-1\right)\left(b_{2}-1\right)}\left(\left(b_{2}-1\right) x+\left(1-a_{1}\right) y\right)\right)^{n} \\
= & \sum_{j=0}^{n}\left(\begin{array}{c}
n \\
j
\end{array}\right) z^{j}\left(\frac{\left(1-a_{3}\right)}{\left(b_{1}-1\right)\left(b_{2}-1\right)}\left(\left(b_{2}-1\right) x+\left(1-a_{1}\right) y\right)\right)^{n-j} \\
= & \left(\frac{\left(1-a_{3}\right)}{\left(b_{1}-1\right)\left(b_{2}-1\right)}\right)^{n}\left(\left(b_{2}-1\right) x+\left(1-a_{1}\right) y\right)^{n} \\
& +\sum_{j=1}^{n}\left(\begin{array}{c}
n \\
j
\end{array}\right) z^{j}\left(\frac{\left(1-a_{3}\right)}{\left(b_{1}-1\right)\left(b_{2}-1\right)}\left(\left(b_{2}-1\right) x+\left(1-a_{1}\right) y\right)\right)^{n-j} \\
= & \left(\frac{\left(1-a_{3}\right)}{\left(b_{1}-1\right)\left(b_{2}-1\right)}\right)^{n}\left(\left(b_{2}-1\right) x+\left(1-a_{1}\right) y\right)^{n}+z g_{2},
\end{aligned}
$$

for some homogeneous polynomial $g_{2}$. Then taking $g_{1}=g_{0}-\tilde{c}_{0} g_{2}$ we obtain (4.8). It follows from (4.8) and the fact that $G^{n}$ is a homogeneous polynomial of degree $n$ with cofactor $-n(x+y+z)$ that

$$
-n(x+y+z)\left(\tilde{c}_{0} G^{n}+z g_{1}\right)=\mathscr{X}\left(\tilde{c}_{0} G^{n}+z g_{1}\right)=-n(x+y+z) \tilde{c}_{0} G^{n}+\mathscr{X}\left(z g_{1}\right),
$$

and so

$$
\mathscr{X}\left(z g_{1}\right)=\mathscr{X}(z) g_{1}+z \mathscr{X}\left(g_{1}\right)=-z\left(a_{3} x+b_{3} y+z\right) g_{1}+z \mathscr{X}\left(g_{1}\right)=-n(x+y+z) z g_{1} .
$$

Hence,

$$
\mathscr{X}\left(g_{1}\right)=\left(-n(x+y+z)+a_{3} x+b_{3} y+z\right) g_{1} .
$$

We will show that $g_{1}=0$. We consider two different cases.

Case 2.1: $g_{1}$ is not divisible by $z$. Let $g_{1}^{*}$ the restriction of $g_{1}$ to $z=0$. Then by assumptions we have that $g_{1}^{*} \neq 0$ and so, $g_{1}^{*}$ is a homogeneous Darboux polynomial of degree $n-1$ of system (3.1) with cofactor $-n(x+y)+a_{3} x+b_{3} y$. It follows from the arguments used in the proof of Theorem 3.1 that

$$
\left(1-b_{2}\right) m_{2}-n=-n+a_{3}, \quad\left(1-a_{1}\right) m_{1}-n=-n+b_{3},
$$

which is not possible because $b_{2}>1$ and $0<a_{3}<1$. So this case is not possible.

Case 2.2: $g_{1}$ is divisible by $z$. We write $g_{1}=z^{\ell} h$ with $\ell$ an integer satisfying $0<\ell<n$ and $h$ a homogeneous polynomial of degree $n-\ell$ that satisfies

$$
\mathscr{X}(h)=\left(-n(x+y+z)+(\ell+1)\left(a_{3} x+b_{3} y+z\right)\right) h .
$$

Let $h^{*}$ be the restriction of $h$ to $z=0$. Then by assumptions of we have that $h^{*} \neq 0$ and so, $h^{*}$ is a homogeneous Darboux polynomial of degree $n-\ell$ of system (3.1) with cofactor $-n(x+y)+(\ell+$ 1) $\left(a_{3} x+b_{3} y\right)$. It follows from the arguments used in the proof of Theorem 3.1 that in this last case we must have

$$
\left(1-b_{2}\right) m_{2}-n=-n+(\ell+1) a_{3}, \quad\left(1-a_{1}\right) m_{1}-n=-n+(\ell+1) b_{3},
$$

which is not possible because $b_{2}>1$ and $0<a_{3}<1$. So this case is not possible. 
In short $g_{1}=0$ and it follows from (4.8) that $f=\tilde{c}_{0} G^{n}$ in contradiction with the fact that $f$ is irreducible because $n>1$. This concludes the proof of the theorem.

Now we prove Theorem 1.3.

Proof of Theorem 1.3. The proof follows directly from Proposition 2.4 and Theorem 4.1.

\section{Proof of Theorem 1.2}

Let $f$ be a homogeneous polynomial first integral of degree $n$. It follows from Theorem 3.1 that $f$ can be written as $f=z h_{1}$ with $h_{1}$ a polynomial of degree $n-1$. Moreover it follows from Theorem 3.2 that $f$ can also be written as $f=x h_{2}$ with $h_{2}$ a polynomial of degree $n-1$ and in view of Theorem 3.3 it can also be written as $f=y h_{3}$ with $h_{3}$ a polynomial of degree $n-1$. Hence, $f$ can be written as

$$
f=z h_{1}, \quad f=x h_{2}, \quad f=y h_{3}
$$

which yields

$$
f=x y z g
$$

where $g$ is a polynomial of degree $n-3$. Taking into account that $\mathscr{X}(f)=0$ we get that

$$
x y z \mathscr{X}(g)-\left(\left(1+b_{2}+a_{3}\right) x+\left(a_{1}+1+b_{3}\right) y+\left(b_{1}+a_{2}+1\right) z\right) x y z g=0
$$

that is, after simplifying by $x y z$,

$$
\mathscr{X}(g)=\left(\left(1+b_{2}+a_{3}\right) x+\left(a_{1}+1+b_{3}\right) y+\left(b_{1}+a_{2}+1\right) z\right) g .
$$

This yields that $g$ is either 0 (which is not possible because then $f=0$ and this is in contradiction with the fact that $f$ is a homogeneous polynomial first integral, or $g$ is a Darboux polynomial. Now taking into account that by Proposition 2.1 any Darboux polynomial factorizes in irreducible Darboux polynomials, by Propositions 2.2 and 2.4 and Theorem 1.3 any first integral (either a polynomial or a proper rational function) must be of the form

$$
f= \begin{cases}x^{m_{1}} y^{m_{2}} z^{m_{3}} & \text { if } b_{3} \neq b_{3}^{*}, \\ x^{m_{1}} y^{m_{2}} z^{m_{3}} G^{m_{4}} & \text { if } b_{3}=b_{3}^{*},\end{cases}
$$

where $m_{1}, m_{2}, m_{3}, m_{4}$ are integers. We consider two different cases.

Case 1: $b_{3} \neq b_{3}^{*}$. In this case we have that $f$ is a polynomial or a proper rational first integral if and only if $f=x^{m_{1}} y^{m_{2}} z^{m_{3}}$, for some integers $m_{1}, m_{2}, m_{3}$ with $m_{1}+m_{2}+m_{3}+m_{4} \neq 0$. Therefore, 


\section{J. Llibre and C. Valls / Proper rational and analytic first integrals}

computing the cofactor $K$ of $f$ and setting it equal to zero we get the equation

$$
0=\left(m_{1}+m_{2} b_{2}+m_{3} a_{3}\right) x+\left(m_{1} a_{1}+m_{2}+m_{3} b_{3}\right) y+\left(m_{1} b_{1}+m_{2} a_{2}+m_{3}\right) z,
$$

that is,

$$
m_{1}+m_{2} b_{2}+m_{3} a_{3}=0, \quad m_{1} a_{1}+m_{2}+m_{3} b_{3}=0, \quad m_{1} b_{1}+m_{2} a_{2}+m_{3}=0 .
$$

This is a linear system in the variables $\left(m_{1}, m_{2}, m_{3}\right)$ that can be written as $A M=0$ where $M=$ $\left(m_{1}, m_{2}, m_{3}\right)$. We compute the determinant of the matrix $A$ and we get

$$
\begin{aligned}
\operatorname{det} A= & 1+a_{1} a_{2} a_{3}-a_{3} b_{1}-a_{1} b_{2}-a_{2} b_{3}+b_{1} b_{2} b_{3} \\
= & 1-a_{2}+a_{2} a_{3}-a_{3} b_{1}-b_{2}+b_{1} b_{2}+a_{1}\left(a_{2} a_{3}-b_{2}\right) \\
& -\left(a_{2} a_{3}-b_{2}\right)+b_{3}\left(b_{1} b_{2}-a_{2}\right)-\left(b_{1} b_{2}-a_{2}\right) \\
= & \left(1-a_{2}\right)\left(1-a_{3}\right)+\left(b_{1}-1\right)\left(b_{2}-a_{3}\right)+\left(b_{3}-1\right)\left(b_{1} b_{2}-a_{2}\right) \\
& +\left(a_{1}-1\right)\left(a_{2} a_{3}-b_{2}\right)>0
\end{aligned}
$$

because $a_{i} \in(0,1)$ and $b_{i}>1$ for $i=1,2,3$. So, the unique solution of (5.2) is $m_{1}=m_{2}=m_{3}=0$, that is not possible.

Case 1: $b_{3}=b_{3}^{*}$. In this case we have that $f$ is as in (5.1), i.e., $f=x^{m_{1}} y^{m_{2}} z^{m_{3}} G^{m_{4}}$ for some integers $m_{1}, m_{2}, m_{3}$ and $m_{4}$ with $m_{1}+m_{2}+m_{3}+m_{4} \neq 0$. Computing the cofactor $K$ of $f$ and setting it equal to zero we get the equation

$$
\left(m_{1}+m_{2} b_{2}+m_{3} a_{3}+m_{4}\right) x+\left(m_{1} a_{1}+m_{2}+m_{3} b_{3}+m_{4}\right) y+\left(m_{1} b_{1}+m_{2} a_{2}+m_{3}+m_{4}\right) z=0 .
$$

Solving $K=0$ in the variables $m_{1}, m_{2}, m_{3}, m_{4}$ we get

$$
\begin{aligned}
& m_{2}=\frac{b_{1}-1}{1-a_{2}} m_{1}, \quad m_{3}=\frac{\left(b_{1}-1\right)\left(b_{2}-1\right)}{\left(1-a_{2}\right)\left(1-a_{3}\right)} m_{1}, \\
& m_{4}=-\frac{1-a_{2}+a_{2} a_{3}-a_{3} b_{1}-b_{2}+b_{1} b_{2}}{\left(1-a_{1}\right)\left(1-a_{3}\right)} m_{1},
\end{aligned}
$$

with $m_{1} \in \mathbb{Z}$. From (1.3) we have $m_{2}=k_{1}^{*} m_{1}, m_{3}=k_{2}^{*} m_{1}$ and $m_{4}=k_{3}^{*} m_{1}$. Now we consider $x y^{k_{1}^{*}} z_{2}^{k_{2}^{*}} G_{3}^{k_{3}^{*}}$. Since $k_{1}^{*}, k_{2}^{*}>0$ and they must be integers, we conclude that $k_{1}^{*}, k_{2}^{*} \in \mathbb{N}$. Moreover, proceeding as in (5.3) we get that

$$
1-a_{2}+a_{2} a_{3}-a_{3} b_{1}-b_{2}+b_{1} b_{2}=\left(1-a_{2}\right)\left(1-a_{3}\right)+\left(b_{1}-1\right)\left(b_{2}-a_{3}\right)>0,
$$

and so $k_{3}^{*}<0$. Since it must be an integer we conclude that $k_{3}^{*}$ is a negative integer. This completes the proof of the theorem.

\section{Acknowledgments}

We thank to the reviewer for his/her helpful comments. The author is partially supported by the FEDER-MINECO grant MTM2016-77278-P, the MINECO grant MTM2013-40998-P, the AGAUR grant number 2014SGR-568. The second author is partially supported by FCT/Portugal through UID/MAT/04459/2013. 


\section{References}

[1] M.A. Almeida, M.E. Magalhães and I.C. Moreira, Lie symmetries and invariants of the Lotka-Volterra system, J. Math. Phys. 36 (1995) 1854-1867.

[2] R.F.S. Andrade and A. Rauh, The Lorenz model and the method of Carleman embedding, Phys. Lett. A 82 (1981) 276-278.

[3] T. Bountis, B. Grammaticos and A. Ramani, On the complete and partial integrability of nonHamiltonian systems, Phys. Rep. 180 (1989) 159.

[4] L. Cairó, H. Giacomini and J. Llibre, Liouvillian first integrals for the planar Lotka-Volterra systems, Rendiconti del circolo matematico di Palermo 53 (2003) 389-418.

[5] F. Cantrijn and W. Sarlet, Generalizations of Noether's theorem in classical mechanics, SIAM Rev. 23 (1981) 467-494.

[6] T. Carleman, Application de la théorie des équations intégrales linéaires aux systémes d'équations différentielles non linéaires, Acta Mathematica 59 (1932) 63.

[7] C. Chi, S. Hsu and L. Wu, On the asymmetric May-Leonard moldel of three competing species, SIAM J. Appl. Math. 58 (1998) 211-226.

[8] G. Darboux, Mémoire sur les équations différentielles algébriques du premier ordre et du premier degré (Mélanges), Bull. Sci. math. 2ème série 2 (1878) 60-96; 123-144; 151-200.

[9] H.J. Giacomini, C.E. Repetto and O.P. Zandron, Integrals of motion of three-dimensional nonHamiltonian dynamical systems, J. Phys. A24 (1991) 4567-4574.

[10] J. Hietarinta, Direct methods for the search of the second invariant, Phys. Rep. 147 (1987) 87-154.

[11] S. Labrunie, On the polynomial first integrals of the $(a, b, c)$ Lotka-Volterra system, J. Math. Phys. 37 (1996) 5539-5550.

[12] P.D. Lax, Integrals of nonlinear equations of evolution and solitary waves, Comm. Pure Appl. Math. 21 (1968) 467-490.

[13] P.G.L. Leach and J. Miritzis, Analytic behavior of competition among three species, J. Nonlinear Math. Phys. 13 (2006) 535-548.

[14] J. Llibre, Integrability of polynoial differential systems, Handbook of Differential Equations, Ordinary Differential Equations, Eds. A. Cañada, P. Drabek and A. Fonda, (Elsevier, 12004 pp. 437-533).

[15] J. Llibre and C. Valls, Global analytic first integrals for the real planar Lotka-Volterra sysetms, J. Math. Phys. 48 (2007) 1-13.

[16] J. Llibre and C. Valls, Polynomial, rational and analytic first integrals for a family of 3-dimensional Lotka-Volterra systems, Z. Angew. Math. Phys. 62 (2011) 761-777.

[17] J. Llibre and X. Zhang, Polynomial first integrals for quasi-homogeneous polynomial differential systems, Nonlinearity 15 (2002) 1269-1280.

[18] J. Llibre and X. Zhang, Darboux theory of integrability in $C^{1}$ taking into account the mulitplicity, $J$. Diff. Equations 246 (2009) 541-551.

[19] R.M. May and W.J. Leonard, Nonlinear aspects of competition between three species, SIAM J. Appl. Math. 29 (1975) 243-256.

[20] J.M. Ollagnier, Polynomial first integrals of the Lotka-Volterra system, Bull. Sci. math. 121 (1997) 463-476.

[21] J.M. Ollagnier, Rational integration of the Lotka-Volterra system, Bull. Sci. math. 123 (1999) 437-466.

[22] J.M. Ollagnier, Liouvillian integration of the Lotka-Volterra system, Qualitative Theory of Dynamical Systems 2 (2001) 307-358.

[23] J.M. Ollagnier, A. Nowicki and J.M. Strelcyn, On the non-existence of derivations: the proof of a theorem of Jouanolou and its developments, Bull. Sci. math. 119 (1995) 195-233.

[24] P.J. Olver, Applications of Lie groups to differential equations, (Springer, New York, 1986).

[25] J.M. Strelcyn and S. Wojciechowski, A method of finding integrals for three-dimensional dynamical systems, Phys. Lett. A 133 (1988) 207-212.

[26] P. Waltman, Competition Models in Population Biology, (SIAM, Philadelphia, PA, 1983). 